\title{
One-dimensional stochastic model of radiative heat transfer in dielectric medium
}

\author{
Yu.P. Virchenko, Lam Tan Phat \\ Belgorod State University, 308015, Belgorod, Russia
}

Received December 16, 2015

\begin{abstract}
One-dimensional stochastic model that describes radiative heat transfer in dielectric medium is built. It is done on the basis of representation that heat transfer in a solid is realized both by means of its heat conductivity and by means of the heat electromagnetic radiation which is generated by thermal fluctuations in the medium. It is supposed that electromagnetic fluctuations are caused by thermal vibrations of solid skeleton connected with irradiation and absorption processes of photons. In the difference with previous works, it permits to formulate the stochastic model without using the supposition of susceptibility fluctuations and not to use the additional mathematical averaging on temporally slow process of thermal conductivity. It leads to the model in the form of the infinite dimensional Ornstein-Uhlenbeck process using the classic fluctuationdissipative theorem. The energy flux of fluctuating electromagnetic field is calculated as a functional of the local temperature.
\end{abstract}

Keywords: stochastic model, radiative heat transfer, fluctuation-dissipative theorem.

Построена стохастическая модель для описания одномерного радиационно-кондуктивного теплообмена в диэлектрической среде. Модель конструируется на основе представления о том, что перенос тепла в твердом теле, в дополнение к его теплопроводности, осуществляется также посредством переноса теплового электромагнитного излучения, порождаемого тепловыми флуктуациями в среде. Предполагается, что флуктуации әлектромагнитного поля вызваны тепловыми колебаниями твердого остова при излучении и поглощении фотонов. Это позволяет, в отличие от предыдущих работ, сформулировать стохастическую модель без использования предположения о флуктуациях электрической восприимчивости и не использовать дополнительное усреднение по медленным тепловым процессам. В результате, на основе флуктуацинно-диссипационной теоремы, получается модель в виде бесконечномерного процесса Орнштейна-Уленбека. Вычисляется поток энергии флуктуационного электромагнитного поля в виде функционала от локальной температуры.

Одновимірна стохастична модель радіаційно-кондуктивного теплообміну. Ю.П.Вірченко, Лам Тан Фат.

Побудовано стохастичну модель для опису одновимірного радіаційно-кондуктивного теплообміну у діелектричному середовищі. Модель утворюється на підставі уявлення, що перенесення тепла у твердому середовищі, у доповненні до його теплопровідності, виконується також внаслідок перенесення теплового электромагнітного випромінювання, що породжується тепловими флуктуаціями у середовищі. Припускається, що флуктуації пов'язані з тепловими коливаннями твердого остову внаслідок випромінювання та поглинання фотонів. Це дозволяє сформулювати стохастичну модель без використання припущення про флуктуації електричної схильності та не використовувати усереднення за повільними тепловими процесами. У результаті на підставі флуктуаційнодисіпаційної теореми одержано модель у вигляді нескінченновимірного процесу Орнштейна-Уленбека. Обчислено струм енергії флуктуаційного электромагнітного поля у вигляді функціоналу від локальної температури. 


\section{Introduction}

Heat transfer in solids is realized by two mechanisms: firstly, via the heat conductivity and, secondly, by the electromagnetic radiation transfer in them which is generated by thermal fluctuations of local thermodynamic state. Accordingly, the evolution equation for the temperature $T(\mathbf{x}, t)$ distribution at the time moment $t$ can be written phenomenologically in the form (see, for example, [1-3].

$$
\rho \vartheta \dot{T}(\mathbf{x}, t)=\varkappa \Delta T(\mathbf{x}, t)-(\nabla, \mathbf{S})(\mathbf{x}, t)
$$

where $\varkappa>0$ is the thermal conductivity coefficient of the medium, $\rho$ is medium density and $\vartheta$ is its heat capacity, where it is supposed that these values do not depend on temperature. The vector field $\mathbf{S}(\mathbf{x}, t)$ represents the energy flux of electromagnetic radiation which is generated by fluctuations of charges and currents induced in the medium. It is assumed that the value $(\nabla, \mathbf{S}(\mathbf{x}, t))$ multiplied to small volume $\Delta V$ of the medium region which is concentrated near the point $\mathrm{x}$, is equal to the part of electromagnetic flux that is spent on heating of this area at the time $t$. Account of this term is significant when heat transfer problems are solved in optically semitransparent media with small electrical conductivity and it is important when there are relatively large temperature differences during the distance being characteristic for the physical situation under consideration. In order to obtain the solution of each heat transfer problem in cases pointed out, it is necessary to obtain a closed evolution equation for $T(\mathrm{x}, t)$. This requires the explicit form of the functional $\mathbf{S}(\mathbf{x}, t)=\mathbf{S}[T(\mathbf{x}, t)]$ which converts Eq. (1) into the self-consistent one.

Usually, the energy flux $\mathbf{S}(\mathbf{x}, t)$ is constructed phenomenologically in the framework of so-called radiative transfer theory. It is done on the basis of geometric optics and Kirchhoff's law about intensities of electromagnetic field radiation and its absorption (see, for example, [1-4]). The thermal electromagnetic field is absent in these constructions. Such a state is unsatisfactory from theoretical point of view. It is connected with the absence of successive microscopic theory of radiative heat transfer in media which should be constituted on the quantum theory both irradiation and absorption of thermal photons in solid-state medium. For this reason, such a theory should be statistical.

Here, we dot not concern to detail analysis of those problems which are necessary to solve when the construction of microscopic theory of radiative heat transfer is built in the framework of statistical physics (see, [5-7]). We point out only that the beginning of statistical approach development in the theory of radiative heat transfer was initiated in the Rytov works which are summarized in the monographs [8,9]. In connection with the complexity at microscopic theory constructing of radiation heat transfer, S.M. Rytov with his collaborators used semi-phenomenological statistical approach. In frameworks of it, the stochastic electromagnetic field is introduced in the theory. At this, the field satisfies Maxwell's equations but any microscopic mechanism of its energy transition into heat is not concretize. It allows to avoid the description of quantum processes of irradiation and absorption that it is reasonable from theoretical point of view since the heat transfer by radiation is not a quantum effect. Stochastic electromagnetic field arises in the theory due to charges thermal fluctuations and electric currents which induced in the medium due to them despite to very small electrical conductivity. Amplitude of these fluctuations increases with the temperature so that at sufficiently large its value, thermal atomic (ionic) vibrations bring to fluctuations of electrical charges on spatial scales of several interatomic distances order in dielectric medium. Average value of them may be essential for the account of generated thermal radiation.

In this work we construct the stochastic model of radiative heat transfer within the frameworks of the described semi-phenomenological approach. In contrast to the model proposed earlier in [5-7], it is based on explicit statistical description of thermal fluctuations of charges and currents in the medium. It is more successive from the theoretical point of view. Because to the complexity of used mathematical structures, we consider only one-dimensional temperature distribution and, consequently, we analyze one-dimensional radiative heat transfer. 


\section{Model construction}

We start using the fact that the thermal electromagnetic field defined by the pair $\{\tilde{\mathbf{E}}(\mathbf{x}, t), \tilde{\mathbf{H}}(\mathbf{x}, t)\}$ at each space-time point $\{\mathbf{x}, t\}$ is the stochastic one (here and after, we denote all random functions by the sign "tilde"). Then, this pair determines the density of the energy flux

$$
\tilde{\mathbf{S}}(\mathbf{x}, t)=\frac{c}{4 \pi}[\tilde{\mathbf{E}}, \tilde{\mathbf{H}}](\mathbf{x}, t)
$$

which is also random field. Thermal electromagnetic field varies rapidly over distances of characteristic wavelength $\left(\sim 10^{-4} \mathrm{~cm}\right)$ order corresponding to thermal (red and infra-red) radiation and, accordingly, during temporal periods about $\sim 10^{-14} \mathrm{~s}$. At the same time, the characteristic time connected with the thermal conductivity process in solid dielectrics should be estimated by the ratio of the squared characteristic length of thermal nonuniformity to the temperature conductivity coefficient. If we take interest the thermal characteristic time for the characteristic nonuniformity of the $1 \mathrm{~cm}$ order, then we have its value $10 \div 10^{2} \mathrm{~S}$ (for example, the $\mathrm{SiO}_{2}$ (quartz) temperature conductivity value $(\varkappa / \rho \vartheta)$ is equal approximately $4 \cdot 10^{-2} \mathrm{~cm}^{2} / \mathrm{s}$, see $\left.[10]\right)$.

Therefore, the density of the energy flux (2) should be averaged over spatial regions having the scale much more greater than the characteristic wavelength of stochastic electromagnetic field, but it is much more smaller than the characteristic length of thermal conductivity process. Besides, it should be also averaged over temporal intervals which have the duration much more greater than the characteristic vibrational period of thermal radiation, but it is much more smaller than the characteristic time of thermal conductivity. Such an averaging permits to take not into account the space and temporal changes of the divergence of radiative flux density $(\nabla, \tilde{S}(\mathbf{x}, t))$ which are very rapid and small, therefore, there is not their influence on the heat transfer process. The pointed out space-time averaging is equivalent to averaging on probability distribution of stochastic electromagnetic field when the field pair $\{\tilde{\mathbf{E}}(\mathbf{x}, t), \tilde{\mathbf{H}}(\mathbf{x}, t)\}$ possesses the ergodicity property. Thus, the field energy flux density that is used in Eq.(1) is determined by the average value $\mathbf{S}(\mathrm{x}, t)=\langle\tilde{\mathbf{S}}(\mathrm{x}, t)\rangle$ (here, angle brackets denote the averaging on the probability distribution). Thus, to formulate the heat transfer problem being closed from the mathematical viewpoint, it is necessary to built an adequate stochastic model of thermal electromagnetic field and to calculate, on its basis, the flux density $\mathbf{S}(\mathbf{x}, t)$ by the averaging pointed out.

The stochastic electromagnetic field is represented by random realizations which satisfy the system of stochastic Maxwell equations in continuum dielectric medium when its dispersion is neglected,

$$
\begin{array}{ll}
\frac{\varepsilon}{c} \frac{\partial \tilde{\mathbf{E}}}{\partial t}+\frac{4 \pi}{c} \tilde{\mathbf{j}}=[\nabla, \tilde{\mathbf{H}}], & (\nabla, \tilde{\mathbf{E}})=\frac{4 \pi}{\varepsilon} \tilde{\rho}, \\
\frac{\mu}{c} \frac{\partial \tilde{\mathbf{H}}}{\partial t}=-[\nabla, \tilde{\mathbf{E}}], \quad(\nabla, \tilde{\mathbf{H}})=0 .
\end{array}
$$

Here, $\tilde{\mathbf{E}}$ и $\tilde{\mathbf{H}}$ are intensities of electrical and magnetic fields of thermal radiation generated by heated medium. At this, $\varepsilon$ and $\mu$ are electric and magnetic permeabilities of uniform dielectric medium, correspondingly, which do not depend on $\{\mathbf{x}, t\}$.

Really, coefficients $\varepsilon=\varepsilon(T)$ and $\mu=\mu(T)$ depend on temperature. Such dependencies are essential at large temperature gradients on distances having the order of characteristic scale of medium sample under consideration. Therefore, generally speaking, it is necessary to take into account in problems of radiative heat transfer when such gradients are present. Since temperature values in dependencies pointed out should be equal to the local temperature $T(\mathbf{x}, t)$, then the Maxwell equations should have spatial and temporal derivatives of $\varepsilon(T(\mathbf{x}, t))$ and $\mu(T(\mathbf{x}, t))$. But, their magnitudes are very small due to pointed out slow dependencies in comparison with scales of length and temporal interval being characteristic of thermal radiation. Therefore, these derivatives we do not taken into account in Eqs.(3).

Random functions $\tilde{\mathbf{j}}, \tilde{\rho}$ in Eqs. (3) are densities of electric current and charge which are generated at microscales of the characteristic wavelength order due to thermal fluctuations. They represent some spatially distributed stochastic sources for Eqs. (3) which are supposed as given ones by the model under consideration. Random realizations of stochastic fields are fully determined by Eqs. (3) if these sources are 
known. As concerns to boundary conditions of the problem which are very important (see, [5-7]), in this work, we consider the simplest situation from physical viewpoint when the dispersal of located thermal inhomogeneity is studied in infinite medium. So, the temperature distribution the medium converges to a constant value when $\mathbf{x}$ goes to infinity.

The equation system (3) is overfull. Therefore, it is necessary the consistency condition for it. It is well-known that such a condition is expressed in the form of the continuity equation

$$
\dot{\tilde{\rho}}+(\nabla, \tilde{\mathbf{j}})=0
$$

and the sources $\tilde{\mathbf{j}}, \tilde{\rho}$ should be submitted to it.

The current density is represented as a sum of stochastic electromagnetic field source (it is an internal "electromotive force" of the medium) which arises due to thermal fluctuations and, besides, the current density induced by electromagnetic field which is expressed in the form of Ohm's law $\sigma \tilde{\mathbf{E}}$. The coefficient $\sigma>0$ plays the role of conductivity in this law, but it is not a genuine macroscopic medium conductivity. It performs an "effective conductivity". It differs from zero due to so-called fuctuation-dissipative theorem (see, for example, [9]). The sense of theorem conjecture is connected with necessity of regular compensating term in stochastic equation with additive noise so that a stationary evolutional regime may exist. In turn, the part of fluctuating current density which serves the stochastic source of electromagnetic field, should contain with probability one the vortical part $u(T(\mathrm{x}, t)) \tilde{\varphi}$ (fluctuating "Foucault currents"), in spite of the fact that charge motion occurs in dielectric medium (or high-resistance semiconductor). Here, $u(T(\mathbf{x}, t))$ is the intensity of the source which depends on the local temperature $T=T(\mathbf{x}, t)$. Thus, this intensity can be varied spatially and temporally, but its variation is more slower in comparison with the thermal electromagnetic fields one. Just the presence of vortical part is connected with the irradiation of electromagnetic waves transferring heat in the medium. In connection with the dielectric medium nature, the fluctuating current (namely, its correlation function) is concentrated at small spatial scales which have the interatomic distance order.

Thus, the density $\tilde{\mathbf{j}}$ should be replaced by $u(T) \tilde{\varphi}+\sigma \tilde{\mathbf{E}}$ in equations (3) and (4). As a result of such a replacement, we obtain the first equation of the system (3) in the form of a stochastic equation with additive noise

$$
\frac{\partial \tilde{\mathbf{E}}}{\partial t}+\gamma \tilde{\mathbf{E}}+\frac{4 \pi}{\varepsilon} u(T) \tilde{\varphi}=\frac{c}{\varepsilon}[\nabla, \tilde{\mathbf{H}}], \quad \gamma=\frac{4 \pi \sigma}{\varepsilon} .
$$

Respectively, Eq. (4) has the form

$$
\dot{\tilde{\rho}}+\gamma \tilde{\rho}+(\nabla, u(T) \tilde{\varphi})=0
$$

where, as in above, we neglect spatial derivatives from the temperature distribution. Here, the coefficient $\sigma$, in general case, should be also depend on the local temperature that is varied slowly with $\mathbf{x}$ and $t$, but we shall also neglect this dependence in stochastic equations (5) and (6) as we already have done.

We consider the random field $\tilde{\varphi}$ as the gaussian one due to its small intensity. Besides, we suppose that it has zero average value $\langle\tilde{\varphi}(\mathbf{x}, t)\rangle=0$. At last, we consider that $\langle\tilde{\rho}(\mathbf{x}, t)\rangle=0$. Then, the field $\tilde{\varphi}$ is fully determined by its pair correlation function $K_{j_{1} j_{2}}\left(\mathbf{x}_{1}-\mathbf{x}_{2}, t_{1}-t_{2}\right)=\left\langle\tilde{\varphi}_{j_{1}}\left(\mathbf{x}_{1}, t_{1}\right) \tilde{\varphi}_{j_{2}}\left(\mathbf{x}_{2}, t_{2}\right)\right\rangle$. Such a form of correlation function means that the field $\tilde{\varphi}(\mathbf{x}, t)$ is stochastically spatially uniform and it is temporally stationary. Then, at the neglect of slow $\mathbf{x}$ and $t$ dependencies of the local temperature, the source $u(T) \tilde{\varphi}(\mathrm{x}, t)$ in Eqs.(5),(6) is a stochastically uniform and stationary random field. Besides, we consider that the field $\tilde{\varphi}(\mathbf{x}, t)$ possesses the property of temporal reversibility. It means that, together with the natural following property $K_{j_{2} j_{1}}\left(\mathbf{x}_{2}-\mathbf{x}_{1}, t_{2}-t_{1}\right)=K_{j_{1} j_{2}}\left(\mathbf{x}_{1}-\mathbf{x}_{2}, t_{1}-t_{2}\right)$, the pair correlation function has the property $K_{j_{1} j_{2}}\left(\mathbf{x}_{1}-\mathbf{x}_{2}, t_{2}-t_{1}\right)=K_{j_{1} j_{2}}\left(\mathbf{x}_{1}-\mathbf{x}_{2}, t_{1}-t_{2}\right)$.

After above-described explicit definition of random process determining the stochastic source $\tilde{\mathbf{j}}(\mathbf{x}, t)$, the fluctuation electromagnetic field is defined completely. Let us introduce generalized Fourier decompositions of random realizations of stochastic fields $\tilde{\mathbf{E}}(\mathbf{x}, t)$ и $\tilde{\mathbf{H}}(\mathbf{x}, t)$,

$$
\tilde{\mathbf{E}}(\mathbf{x}, t)=\int_{\mathbb{R}^{3}} \tilde{\tilde{\mathbf{E}}}(\mathbf{k}, t) \exp [i(\mathbf{k}, \mathbf{x})] d \mathbf{k}, \quad \tilde{\mathbf{H}}(\mathbf{x}, t)=\int_{\mathbb{R}^{3}} \tilde{\mathbf{H}}(\mathbf{k}, t) \exp [i(\mathbf{k}, \mathbf{x})] d \mathbf{k} .
$$


Here, $\tilde{\overline{\mathbf{E}}}(\mathbf{k}, t)$ and $\tilde{\overline{\mathbf{H}}}(\mathbf{k}, t)$ are generalized random fields relative to $\mathbf{k} \in \mathbb{R}^{3}$. Further, we substitute decompositions (7) in Eqs. (3), (5), (6). Then, due to one-valuedness of their definition at each $\mathbf{k} \in \mathbb{R}^{3}$ on the basis of the Fourier decompositions, we obtain the finite equation system for generalized Fourier transforms,

$$
\begin{aligned}
& \frac{\partial}{\partial t} \tilde{\overline{\mathbf{E}}}(\mathbf{k}, t)+\gamma \tilde{\tilde{\mathbf{E}}}(\mathbf{k}, t)+\frac{4 \pi \tilde{\mathbf{j}}}{\varepsilon}(\mathbf{k}, t)=\frac{i c}{\varepsilon}[\mathbf{k}, \tilde{\mathbf{H}}(\mathbf{k}, t)], \\
& \frac{\partial}{\partial t} \tilde{\overline{\mathbf{H}}}(\mathbf{k}, t)=-\frac{i c}{\mu}[\mathbf{k}, \overline{\tilde{\mathbf{E}}}(\mathbf{k}, t)], \quad(\mathbf{k}, \tilde{\overline{\mathbf{E}}}(\mathbf{k}, t))=-\frac{4 \pi i}{\varepsilon} \tilde{\bar{\rho}}(\mathbf{k}, t), \quad(\mathbf{k}, \tilde{\overline{\mathbf{H}}}(\mathbf{k}, t))=0, \\
& \dot{\tilde{\rho}}(\mathbf{k}, t)+\gamma \tilde{\rho}(\mathbf{k}, t)+i(\mathbf{k}, \tilde{\overline{\mathbf{j}}}(\mathbf{k}, t))=0
\end{aligned}
$$

where generalized Fourier transforms of charge distribution density realizations are introduced

$$
\tilde{\rho}(\mathbf{x}, t)=\int_{\mathbb{R}^{3}} \tilde{\bar{\rho}}(\mathbf{k}, t) \exp [i(\mathbf{k}, \mathbf{x})] d \mathbf{k}
$$

and also generalized Fourier transforms $\tilde{\overline{\mathbf{j}}}(\mathbf{k}, t)$ of random field realizations $u(\mathbf{x}, t) \tilde{\varphi}(\mathbf{x}, t)$ are defined by the relation

$$
u(T(\mathbf{x}, t)) \tilde{\boldsymbol{\varphi}}(\mathbf{x}, t)=\int_{\mathbb{R}^{3}} \tilde{\mathbf{j}}(\mathbf{k}, t) \exp [i(\mathbf{k}, \mathbf{x})] d \mathbf{k} .
$$

The field $\tilde{\overline{\mathbf{j}}}(\mathbf{k}, t)$ is the complex-valued gaussian random one due to the field $\tilde{\varphi}(\mathbf{x}, t)$ is gaussian. It has the zero average value $\langle\tilde{\mathbf{j}}(\mathbf{k}, t)\rangle=0$. Due to the field $u(T(\mathbf{x}, t)) \tilde{\varphi}(\mathbf{x}, t)$ is real-valued, realizations of the field $\tilde{\mathbf{j}}(\mathbf{k}, t)$ have the property $\tilde{\mathbf{j}}(\mathbf{k}, t)=\tilde{\mathbf{j}}(-\mathbf{k}, t)$ with the probability one. Therefore, this filed is characterized completely by the correlation function $\bar{K}_{j_{1} j_{2}}\left(\mathbf{k}_{1}, t_{1} ; \mathbf{k}_{2}, t_{2}\right)=\left\langle\tilde{\bar{\varphi}}_{j_{1}}\left(\mathbf{k}_{1}, t_{1}\right) \tilde{\bar{\varphi}}_{j_{2}}^{*}\left(\mathbf{k}_{2}, t_{2}\right)\right\rangle$ which is represented by a positive matrix-function on $\mathbf{k} \in \mathbb{R}^{3}$ and $t$.

Since the equation system is finite at each fixed $\mathbf{k} \in \mathbb{R}^{3}$, it is solved uniquely at given initial conditions of generalized random realizations of the fields $\tilde{\mathbf{E}}(\mathbf{k}, t), \tilde{\mathbf{H}}(\mathbf{k}, t), \tilde{\bar{\rho}}(\mathbf{k}, t)$. It means that the stochastic model of thermal electromagnetic field described in this section is self-consistent from the mathematical viewpoint.

\section{Thermal radiation at quasistationary regime}

Due to linearity of the equation system that determine generalized fields $\tilde{\mathbf{E}}(\mathbf{k}, t)$ and $\tilde{\mathbf{H}}(\mathbf{k}, t)$, the electromagnetic field is the gaussian random one with zero average value, if the average value of the filed $\tilde{\bar{\rho}}(\mathbf{k}, t)$ is also equal to zero.

The initial conditions of mathematical expectations of all possible functions constructed on the basis of the fields $\tilde{\mathbf{E}}(\mathbf{k}, t), \tilde{\mathbf{H}}(\mathbf{k}, t)$ and $\tilde{\bar{\rho}}(\mathbf{k}, t)$ are become inessential after a temporal period being much more than the characteristic time $\tau$ that associates with these fields. The time $\tau$ is determined so that the value $\hbar \tau^{-1}$ has the order of medium average temperature multiplied to Boltzman's constant $\kappa$. Since the field $\tilde{\varphi}(\mathbf{x}, t)$ is temporally stationary, then the stochastic fields $\{\tilde{\mathbf{E}}(\mathbf{k}, t), \tilde{\mathbf{H}}(\mathbf{k}, t)\}$ submitted to Eqs. (8)-(10) one may consider as stationary random processes, if we neglect the temporal dependence of the temperature distribution $T(\mathbf{x}, t)$ in the amplitude $u(T(\mathbf{x}, t))$. In such a situation, it is naturally to pass from evolution Eqs. (8)-(10) to equations for spectral decomposition amplitudes of corresponding fields which are generalized functions on the frequency $\omega$ (if we do not take into account the so-called singular constituent of the spectral measure (see, [11, 12]),

$$
\begin{aligned}
\tilde{\overline{\mathbf{E}}}(\mathbf{k}, t) & =\int_{-\infty}^{\infty} \tilde{\mathcal{E}}(\mathbf{k}, \omega) e^{i \omega t} d \omega, \quad \tilde{\mathbf{H}}(\mathbf{k}, t)=\int_{-\infty}^{\infty} \tilde{\mathcal{H}}(\mathbf{k}, \omega) e^{i \omega t} d \omega, \\
\tilde{\overline{\mathbf{j}}}(\mathbf{k}, t) & =\int_{-\infty}^{\infty} \tilde{\iota}(\mathbf{k}, \omega) e^{i \omega t} d \omega, \quad \tilde{\bar{\rho}}(\mathbf{k}, t)=\int_{-\infty}^{\infty} \tilde{\varrho}(\mathbf{k}, \omega) e^{i \omega t} d \omega .
\end{aligned}
$$


Substituting those decompositions into Eqs. (8-10) and using the single-valuedness of Fourier transforms, we obtaine the self-consistent system of equations:

$$
\begin{gathered}
i \omega \tilde{\mathcal{E}}(\mathbf{k}, \omega)+\gamma \tilde{\mathcal{E}}(\mathbf{k}, \omega)+\frac{4 \pi}{\varepsilon} \tilde{\imath}(\mathbf{k}, \omega)=\frac{i c}{\varepsilon}[\mathbf{k}, \tilde{\mathcal{H}}(\mathbf{k}, \omega)], \\
\tilde{\mathcal{H}}(\mathbf{k}, \omega)=-\frac{c}{\mu \omega}[\mathbf{k}, \tilde{\mathcal{E}}(\mathbf{k}, \omega)], \quad(\mathbf{k}, \tilde{\mathcal{E}}(\mathbf{k}, \omega))=-\frac{4 \pi i}{\varepsilon} \tilde{\varrho}(\mathbf{k}, \omega), \quad(\mathbf{k}, \tilde{\mathcal{H}}(\mathbf{k}, \omega))=\mathbf{0}, \\
i \omega \varrho \tilde{\varrho}(\mathbf{k}, \omega)+\gamma \tilde{\varrho}(\mathbf{k}, \omega)+i(\mathbf{k}, \tilde{\iota}(\mathbf{k}, \omega))=\mathbf{0},
\end{gathered}
$$

Solutions of this system in terms of Fourier-transforms $\tilde{\mathcal{E}}(\mathbf{k}, \omega)$ and $\tilde{\mathcal{H}}(\mathbf{k}, \omega)$ are defined by:

$$
\begin{gathered}
\tilde{\mathcal{E}}(\mathbf{k}, \omega)=i \frac{4 \pi}{\varepsilon} \cdot \frac{\left(\left(\omega^{2}-i \omega \gamma\right) \tilde{\imath}(\mathbf{k}, \omega)-\bar{c}^{2}(\mathbf{k}, \tilde{\iota}(\mathbf{k}, \omega)) \mathbf{k}\right)}{(\omega-i \gamma)\left(\omega^{2}-\bar{c}^{2} \mathbf{k}^{2}-i \omega \gamma\right)}, \\
\tilde{\mathcal{H}}(\mathbf{k}, \omega)=-i \frac{4 \pi c}{\varepsilon \mu} \cdot \frac{[\mathbf{k}, \tilde{\iota}(\mathbf{k}, \omega)]}{\left(\omega^{2}-\bar{c}^{2} \mathbf{k}^{2}-i \omega \gamma\right)} .
\end{gathered}
$$

\section{One-dimensional heat radiation transfer}

We calculate now the flux density of thermal radiation on the basis of the obtained formulas. We restrict our study by one-dimensional radiative heat transfer. It is not in contradiction with the fact that thermal electromagnetic field should has three directions of electromagnetic waves propagation in the sample. The restriction pointed out is associated, firstly, with the fact that, in multidimensional case, there is a great variety of supplement conditions of heat transfer which one should take into account. They are sequence, firstly, of strong dependence on the medium sample geometry, and, secondly, due to necessity of a much more complicated analysis connected with the origin of generalized functions contain a strong singularity. In one-dimensional case, the function $u(\mathbf{x}, t)$ depends only on one coordinate $x, u(\mathbf{x}, t) \equiv u(x, t)$ and, accordingly, we suppose that the field $\tilde{\varphi}(x, t)$ depends on this coordinate only. Then, the wave vector $\mathbf{k}$ has only one nonzero component $k$ and it is directed along the $x$-axis. In accordance with this fact, the Fourier decompositions (7) are turned out from three-dimensional forms to one-dimensional ones. For the calculation of flux density $S(x, t)$ that depends only from one coordinate $x$ and it is directed along the $x$-axis, we use the formulas obtained in the previous section, taking into account the above described changes. Substituting Eq.(18) and Eq.(19), firstly, into Eq.(13), further, into Eq. (7) and, finally, into Eq.(2), we find:

$$
\begin{gathered}
S(x, t)=\int_{\mathbb{R}^{4}} Q\left(x-y_{1}, t-s_{1} ; x-y_{2}, t-s_{2}\right) K\left(y_{1}-y_{2} ; s_{1}-s_{2}\right) u\left(y_{1}, s_{1}\right) u\left(y_{2}, s_{2}\right) d y_{1} d y_{2} d s_{1} d s_{2}, \\
Q\left(y_{1}, s_{1} ; y_{2}, s_{2}\right)= \\
-\frac{4 \pi c^{2}}{\varepsilon^{2} \mu}\left\{i \dot{J}\left(y_{1}, s_{1}\right) V^{\prime}\left(y_{2}, s_{2}\right)+\dot{V}\left(y_{1}, s_{1}\right) V^{\prime}\left(y_{2}, s_{2}\right)-i \bar{c}^{2} W^{\prime \prime}\left(y_{1}, s_{1}\right) V^{\prime}\left(y_{2}, s_{2}\right)\right\}, \\
K(x-y ; t-s)=\frac{1}{3} K_{l l}(x-y ; t-s)
\end{gathered}
$$

where the functions $J(x, t), V(x, t), W(x, t)$ are "thermal radiation propagators" which are defined by integral representations and they are some generalized functions

$$
\begin{gathered}
J(x, t)=\frac{1}{(2 \pi)^{2}} \int_{\mathbb{R}^{2}} \frac{\exp (i k x+i \omega t)}{\omega-i \gamma} d k d \omega=i \delta(x) \Theta(t) e^{-\gamma t} \\
V(x, t)=\frac{1}{(2 \pi)^{2}} \int_{\mathbb{R}^{2}} \frac{\exp (i k x+i \omega t)}{\omega^{2}-\bar{c}^{2} k^{2}-i \omega \gamma} d k d \omega
\end{gathered}
$$




$$
W(x, t)=\frac{1}{(2 \pi)^{2}} \int_{\mathbb{R}^{2}} \frac{\exp (i k x+i \omega t)}{(\omega-i \gamma)\left(\omega^{2}-\bar{c}^{2} k^{2}-i \omega \gamma\right)} d k d \omega .
$$

The functions $V(x, t)$ and $W(x, t)$ are not calculated strictly. We have evaluated their asymptotical views according to the parameter $\gamma L / \bar{c}$ where $L$ is the characteristic spatial size of thermal uniformity, taking into account that the light velocity $\bar{c}$ in semitransparent medium is very large. It is important that there are the relations $V^{*}(x, t)=V(x, t)$, $W^{*}(x, t)=-W(x, t)$ for them. Calculate integrals in each formulas (23), (24) defined these functions. Firstly, we do it integrating over $\omega$ on the basis of residuals in the poles $\omega=i \gamma, \omega=i \gamma / 2 \pm \omega(k)$, $\omega(k)=\left(k^{2} \bar{c}^{2}-(\gamma / 2)^{2}\right)^{1 / 2}$ which are in the upper half-plane, we obtain

$$
V(x, t)=-\frac{\Theta(t)}{2 \pi} e^{-\gamma t / 2} \int_{\mathbb{R}} e^{i k x} \frac{\sin (\omega(k) t)}{\omega(k)} d k
$$

or, with the account of the above mentioned neglect by terms being small on the parameter $\gamma L / \bar{c}$, we have

It gives

$$
V(x, t)=-\frac{\Theta(t)}{4 \bar{c}} e^{-\gamma t / 2}(\operatorname{sgn}(x+\bar{c} t)-\operatorname{sgn}(x-\bar{c} t))
$$

$$
\begin{aligned}
& \dot{V}(x, t)=-\frac{\gamma}{2} V(x, t)-\frac{\Theta(t)}{4} e^{-\gamma t / 2}(\delta(x+\bar{c} t)+\delta(x-\bar{c} t)) \\
& V^{\prime}(x, t)=-\frac{\Theta(t)}{4 \bar{c}} e^{-\gamma t / 2}(\delta(x+\bar{c} t)-\delta(x-\bar{c})) .
\end{aligned}
$$

Further, we find also by the same way

$$
W^{\prime \prime}(x, t)=\frac{i}{\bar{c}^{2}} \Theta(t)\left\{e^{-\gamma t} \delta(x)-\frac{1}{2} e^{-\gamma t / 2}\left[\delta(x+\bar{c} t)+\delta(x-\bar{c} t)-\frac{\gamma}{4 \bar{c}}(\operatorname{sgn}(x+\bar{c} t)-\operatorname{sgn}(x-\bar{c} t))\right]\right\}
$$

For the calculation of the energy flux density $S(x, t)$, we use the transition to the limit of very short correlation lengths of induced currents, $K(x, t) \rightarrow K(0) \delta(x) \delta(t)$, that is substantiated by the physical circumstance that, in dielectrics, one may use representation about such stochastic currents only which show itself at interatomic distances and during temporal intervals with average value order corresponding to thermal vibrations. It is important to do such a transition after the fulfilling of all integrations in Eq.(20) on the basis of $\delta$-functions properties. It is necessary in order to do the regularization of a product of generalized functions with coincided singularities.

Substitution the expressions (25), (26) into Eq.(21) and, further, into Eq.(20) leads us to the following formula of the energy flux density after calculation of all integrals and transition to the limit of short correlation lengths

$$
S(x, t)=\nu \frac{d}{d x} \int_{\mathbb{R}} e^{-\gamma|y-x| / \bar{c}} u^{2}(y, t-|x-y| / \bar{c}) d y, \quad \nu=\frac{3 \pi \bar{c} K(0)}{4 \gamma \varepsilon} .
$$

According to supposition mentioned in third section, $u(x, t)$ depends on coordinates and time through the functional dependence on the temperature distribution $u(x, t)=u(T(x, t))$. At this, $u^{2}(x, t)$ has the physical sense of the energy density of thermal photon gas at the temperature $T(x, t)$ in the space point $x$ and at the time moment $t$. This value is calculated on the basis of statistical representations of the photon energy $\hbar \omega$ average value with the frequency $\omega$ using the distribution function $f(\hbar \omega / \kappa T(x, t))(\kappa$ is the Boltzman constant). Namely, we set

$$
u^{2}(x, t)=\hbar \int \omega^{3} f(\hbar \omega / \kappa T(x, t)) d \omega
$$

where, in particular, one may put the Plank distribution function as the function $f(\hbar \omega / \kappa T(x, t))$. 


\section{Conclusion}

After the neglect of the time-lag in the formula (27), the obtained expression of energy flux density coincides to within designations with the expression of this value which is obtained from the standard theory of the radiative transfer theory (see, [1-3])

$$
S(x, t)=\nu \frac{d}{d x} \int_{\mathbb{R}} e^{-\gamma|y-x| / \bar{c}} u^{2}(y, t) d y .
$$

Such a neglect is substantial because of the time-lag is extremely small in comparison with the characteristic time corresponding to heat conductivity that occurs at distances of the thermal inhomogeneity order (it has the order of the real sample size).

One may say that despite to the applied value of classical radiation transfer theory, at present time, there is not anything consequential theoretic microscopic approach to the study of thermal radiation transfer in condensed media. Such an approach should be based on statistical physics representations. As it is mentioned above in the introduction, Rytov's works are the pioneer ones along this scientific direction. It has set the question about the development of such a microscopic theory. An attempt has been undertaken in works of an author of proposed article where thermal electromagnetic field has been introduced by explicit way into theoretical model construction. But, the model proposed in mentioned works possesses two essential defects from theoretical viewpoint. Firstly, the damping of thermal fluctuations being necessary for the stabilization of thermal electromagnetic field spreading has been introduced in it by an artificial way. Secondly, the separation of the slow process of temperature distribution change form the fast process of passing to a stationary regime of electromagnetic field is not fulfilled by explicit way in the model. At present work, it is formulated the stochastic model without using of the supposition about electrical susceptibility fluctuations that permits to introduce the damping on the basis of fluctuation-dissipation theorem. Besides, we do not use the additional averaging on temporal period that is very large in comparison with the stabilization time connected with thermal irradiation spreading in the media. At this point of view, the model construction and its verification which have been fulfilled in the work, presents an achievement of the radiative heat transfer theory along with the model studied in [5-7]. It is taken place because our theory clarifies what approximations are necessary in order one may use the traditional theory of radiative heat transfer, and, besides, it connects macroscopic characteristics of radiative heat transfer (the optical length) with statistical characteristics of thermal microcurrent fluctuations. It is important for theory development in the future.

\section{References}

1. E.M. Sparrow, R.D. Cess, Radiation Heat Transfer / Brooks/Cole Publishing Company, Belmont, California (1970).

2. N.A. Rubtsov, Radiation Heat Transfer in Continuos Media / Nauka, Siber Department, Novosibirsk (1984) [in Russian].

3. V.A. Petrov, N.V. Marchenko, Radiation Energy Transfer in Partially Transparent Solid Materials, Nauka, Moscow (1985) [in Russian].

4. A.V. Kolesnikov, Yu.P. Virchenko, Functional Materials, 13, 372, 2006.

5. Yu.P. Virchenko, M.A. Saprykin, Sci. Bull.Belgorod State University. Mathematics \& Physics, 16 (60), 7, 2009 [in Russian].

6. Yu.P. Virchenko, M.A. Saprykin, Functional Materials, 18, 504, 2011.

7. Yu.P. Virchenko, M.A. Saprykin, Reports of NASU, 12, 63, 2010[in Russian].

8. S.M. Rytov. Theory of Electrical Fluctuations and Thermal Radiation. AN SSSR Publishing, Moscow (1953) [in Russian].

9. S.M. Rytov, Yu.A. Klyatskin, V.I. Tatarskii. Introduction to Statistical Radiophysics, 2. Random fields. "Nauka Moscow (1978) [in Russian].

10. Acoustic crystals. Reference book / Ed. M.P.Shaskolskaya / "Nauka Moscow (1982) [in Russian].

11. A.M. Yaglom, Mat.Mathem. Congress, V.1 / Leningrad University, Leningrad, - P.250 (1963) [in Russian].

12. I.I. Gikhman, A.V. Skorokhod, Theory of Random Processes, 1, Nauka, Moscow, 1971 [in Russian]. 